\title{
EFFECT OF DIFFERENT TECHNIQUES OF CAD/CAM DESIGNED BIOHPP FRAMEWORKS ON THE SUPPORTING STRUCTURES OF KENNEDY CLASS I TELESCOPIC PARTIAL DENTURE CASES
}

\author{
Heba Refaat Elsarrif*, Shaimaa Lotfy Mohamed** and Marwa Ezzat Sabet***
}

\begin{abstract}
Objective: This study was carried out to radiographically evaluate the effect of the two different CAM techniques of BioHpp frameworks fabrication (milled and pressed) on the supporting structures of Kennedy Class I telescopic partial dentures using the digital parallel radiographic technique ( Digora software system).

Materials and Methods: Fourteen patients with mandibular Kennedy class I and opposing short maxillary Kennedy class I were selected. The last standing abutments for all patients were the premolars. After receiving zirconia primary crowns, patients were divided into two groups, group I received milled BioHpp telescopic RPDs, and group II received pressed BioHpp telescopic RPDs. Patients of both groups were evaluated at the time of denture insertion, at six months, and 12 months. The evaluation included measurements of bone height changes mesial of the first abutment and distal of the second abutment and at the crest of the residual ridge.
\end{abstract}

Results: In this study, at the end of follow up period, there was statistically significant difference in the marginal bone height changes between the two groups. Group I (milled BioHpp) showed more bone resorption around the abutments than group II, while group II (pressed BioHpp) showed more resorption at the crest of the residual ridge than group I.

Conclusion: Within the limitation of this study, it could be concluded that, milled and pressed BioHpp telescopic partial denture produced acceptable treatment options regarding bone loss. Milled BioHpp frameworks (group I) had a greater effect on bone resorption around the abutments than pressed BioHpp (group II) while at the residual ridge area, pressed BioHpp showed more bone height changes than the milled one.

KEYWORDS: CAD/CAM; PEEK; Zirconia crowns; Telescopic RPD; Bone Evaluation.

\footnotetext{
* Assistant Lecturer in Prosthodontics Department, $6^{\text {th }}$ of October University

** Assistant Professor of Prosthodontics, Ain Shams University.

*** Professor of Prosthodontics, Ain Shams University.
} 


\section{INTRODUCTION}

Rehabilitation of mandibular bilateral distal extension cases has been always a challenging situation. Thus, the provision of a stable, retentive, and biomechanically acceptable removable partial denture is mandatory to preserve the remaining abutments and residual supporting structures. ${ }^{(1)}$

Over the years, there have been several treatment approaches aimed at finding an adequate distribution of occlusal forces between the natural teeth and the residual ridge. As a result, various types of clasps, precision, semi-precision attachments, and telescopic crowns have been used to secure the distal extension of the removable partial denture and to regulate the excessive torquing forces acting on the abutment and to maintain the abutment teeth and their associated supporting structures. Telescopic denture is a type of prosthodontic therapy where its main goal is to reduce occlusal forces from the artificial teeth on the abutment by transferring those forces to the abutment's long axis with the benefit of incorporating proprioception since the periodontal fibres of the retained teeth provide patients with this sense of pressure. ${ }^{(2,3)}$

The direction nowadays is to provide patients with non-metallic restorations that are aesthetically pleasing and could have the same strength and durability as metallic restorations. With the advent of CAD/CAM technology and the evolution in ceramic materials as well as partial denture materials. ${ }^{(4,5)}$ It was appealing in the current study to combine zirconia as a primary crown and polyether-ether-ketone (PEEK) as a secondary crown that was merged in a partial denture framework made from the same material.

PEEK is a recently introduced material for removable partial denture frameworks, and BioHpp material is considered a high-performance polymer of PEEK that is reinforced with ceramic fillers. The BioHPP is supplied in three different forms (blanks, granules, and pellets), and is fabricated using two methods which are milling using dental milling machines and pressing, using a specially designed vacuum press machine. ${ }^{(6)}$

Several in-vitro studies have been performed on zirconia and PEEK as a combined double crown system, ${ }^{(7,8,9)}$ however, few clinical investigations were conducted to evaluate their effect on the supporting structures of tooth-supported partial overdentures.

Also, the existence of two techniques for manufacturing the Bio-HPP restorations might raise the question of which technique is better, in terms of product accuracy, technical difficulties, and advantages to the supporting structures. Thus, this clinical study was conducted to evaluate if the difference between the two techniques (milling and pressing) has affected the supporting structures in mandibular Kennedy Class I telescopic partial denture cases.

\section{MATERIALS AND METHODS}

This clinical trial was approved by the Prosthodontic Department Board and Ethics Committee of the Faculty of Dentistry, Ain Shams University. The study protocol has been approved by the faculty Ethics Committee under the number (FDASU-RecID021719)

\section{Inclusion / exclusion criteria for the participants}

Fourteen partially edentulous patients with Kennedy class I classification were recruited from the outpatient clinic of the Prosthodontics Department, Faculty of Dentistry, Ain Shams University during the period from July 2017 to March 2020. The inclusion criteria for this study involved patients who were (1) aged from 35 to 50 and were able to provide informed consent. (2) Patients with mandibular Kennedy class I, with lower premolars as last standing abutments. (3) Patients with maxillary Kennedy class I distal extensions.(4) Patients with functionally normal occlusion, normal maxilla-mandibular relationship, and enough inter-arch space $[\geq 15 \mathrm{~mm}]$. (5) The 
remaining teeth were free from any periodontal diseases. (6) The distal extension residual alveolar ridge possessed adequate bony support, and free from any pathologic signs, bony undercuts, remaining roots, or neoplastic lesions. (7) The distal extension alveolar ridge covered by healthy mucosa of even thickness and free of inflammation (8) Patients with good oral hygiene.

Exclusion criteria: (1) Patients with parafunctional habits and temporomandibular problems. (2) Patients received or underwent radiotherapy or chemotherapy or had any systemic disease that might affect bone metabolism. (3) Vulnerable groups (mental disorders, patients not capable of decision making, narcotic drug addicts). (4) Uncooperative patients who had no understanding of the need for a regular follow up.

\section{Patient examination}

An initial evaluation was conducted to determine whether the patient met the study inclusion criteria or not. This evaluation consisted of a medical history questionnaire, clinical examination, and radiographic assessment.

\section{Informed consent}

All patients were asked to sign an informed consent form, which was translated into Arabic so that the patients could understand it.

\section{Interventions and study procedures}

An upper flexible removable partial denture was constructed for all patients following the conventional steps. Also, patients received zirconia primary crowns after performing elective endodontic treatment to create enough space for the double crowns on the lower selected abutments before they were divided into two groups.

\section{a) Patient grouping (randomization process):}

Regarding random sequence generation, after construction of the upper flexible removable partial denture, the fourteen patients were assigned randomly into two equal groups, each containing seven patients, using a research randomizer (https:// www.randomizer.org/). Group I (intervention group) included seven mandibular Kennedy class I partially edentulous patients who went on to receive a milled BioHpp telescopic partial denture. Group II (intervention group) included seven mandibular Kennedy class I partially edentulous patients who went on to receive a pressed BioHpp telescopic partial denture.

Regarding treatment allocation, only one investigator, who was not involved in patient selection or treatment, was aware of the randomization sequence and had access to the randomization lists stored on a password-protected laptop. The randomly generated codes were placed in sequentially ordered, opaque, and sealed identical envelopes. Patients were asked to choose one of the envelopes; the investigator, who was informed of the randomization process, was then asked to indicate the group, and the patient was treated as such.

\section{b) Blinding:}

The care provider was advised to avoid discussing treatments available in the subject's presence, and an independent assessor who was not acquainted with the type of intervention evaluated the radiographic periapical images.

\section{c) Mouth preparation:}

Reduction of mandibular premolars with chamfer finish line of $1.5 \mathrm{~mm}$, and axial reduction of $2 \mathrm{~mm}$, and occlusal reduction of $2 \mathrm{~mm}$ was created; the finish line was placed equigingival. The axial walls were with approximately $6^{\circ}$ to $8^{\circ}$ taper. An impression of the preparations was made using polyvinyl siloxane rubber base impression material (Elite HD plus; Zhermack) this was followed by a jaw relation record using the wax wafer technique (Modelling wax; Cavex). Master casts (BegoStone; BEGO) were then obtained and prepared for scanning (Identica Hybrid; Medit, Korea) and construction of the primary zirconia crowns. 


\section{d) Designing and construction of the primary copings}

The primary copings were designed using the CAD software (exocad; Exocad $\mathrm{GmbH}$ ). The same parameters for designing primary copings were maintained for the two groups, the mesial and the distal surfaces with zero degrees taper, the buccal and the lingual surfaces with three degrees of taper. The cement gap was 20 microns except an area of $0.5 \mathrm{~mm}$ from the margins was with no cement gap. The zirconia copings were designed to be 0.5 $\mathrm{mm}$ in thickness. (Figure 1) The standard tessellation language (STL) file of the primary copings was sent to the CAM software (vhf camfacture AG) for nesting and preparation of the zirconia primary copings (Z-CAD; Metoxit) to be milled, in a dry 5-axis dental milling machine (K5; vhf camfacture AG). After that it was verified intra-orally to check marginal adaptation. Next, jaw relation and facebow (A7 PLUS; Bioart) records were done, then pick-up impression of the copings was made with full borders extension within the physiologic limits of the muscles. In the laboratory, a resin pattern (Pattern Resin LS; GC) was placed inside the zirconia copings after lubricating its internal surfaces, then stone was poured inside the whole impression.

\section{e) Designing of the secondary crowns:}

The zirconia copings were placed onto the cast and were sprayed (scan spray; Shera) before scanning, later, the CAD software was used to design the secondary copings alone.

A circumferential play of 35 microns was set between the inner and the outer crowns. The secondary crowns were designed with labial cutbacks.

\section{f) Designing of the partial denture framework:}

The partial denture framework was designed using the partial-cad module, which is an add-on module in the Exocad software, as following: model was surveyed, and undercuts been determined. All unfavourable undercuts were blocked out by the software and a modified cast was created. Denture base meshwork was then outlined. Then the outline of the lingual plate major connector was drawn. External finish line between denture base meshwork and major connector was then drawn. The final design was then merged with the secondary crowns design. For both groups, the final design was sent to the CAM software to be initially milled from castable polymethyl methacrylate resin (PMMA disks, Yamahachi Dental), for intra-oral verification.

\section{g) Manufacturing of the final telescopic partial denture framework from BioHpp material:}

Group I: The framework STL file was sent to the CAM software to prepare the milling machine order for milling the framework directly from the BioHpp blank (BreCAM.BioHPP, Bredent $\mathrm{GmbH}$ ) (Figure 2-A), the milled framework was then sent for intraoral inspection. Finally, the heat-cured acrylic resin (Vertex Rapid Simplified; Vertex) denture base was packed and cured to the denture base meshwork as conventional, then the composite resin (Crea. lign Bredent $\mathrm{GmbH}$ ) was added following the teeth shade on the labial aspect of the secondary crowns following the manufacturer's bonding system protocol (Visiolink, Bredent GmbH). (Figure 3)

Group II, the STL file of the designed framework was firstly milled from the PMMA castable resin, then the resin framework was sprued and placed in a suitable muffle, then the investment material was mixed in a vacuum mixer following the manufacturer recommended ratios, and then poured carefully under vibration inside the muffle. Later, the muffle and the press plunger were placed in a preheated furnace at $850^{\circ} \mathrm{C}$ for one hour, then left for another one hour at that temperature to cool down till it reaches $400^{\circ} \mathrm{C}$. After a waiting period of 20 minutes at this temperature, the BioHpp granules (BioHpp Granules; Bredent $\mathrm{GmbH}$ ) were filled into the muffle and left for 20 minutes. The muffle with the melted BioHpp granules and the plunger were placed onto the pressing table of the device, then vacuum press process with a pressure of 4.5 bar ran automatically for 3 minutes. Next, the framework 
was divested using $110 \mu \mathrm{m}$ alumina particles, then it was trimmed and polished as preparation for intraoral inspection. (Figure 2-B) The heat-cured acrylic resin denture base was then added to the denture base meshwork and fabricated as conventional, finally, the composite resin was added on the labial aspect of the secondary crowns as described with group I.

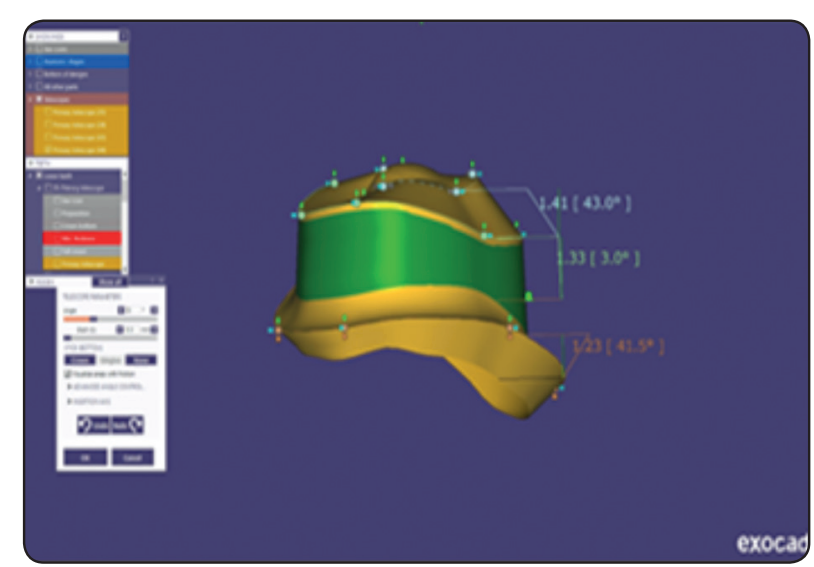

Fig. (1) (a-b): a) virtual primary copings. b) virtual telescopic removable partial denture

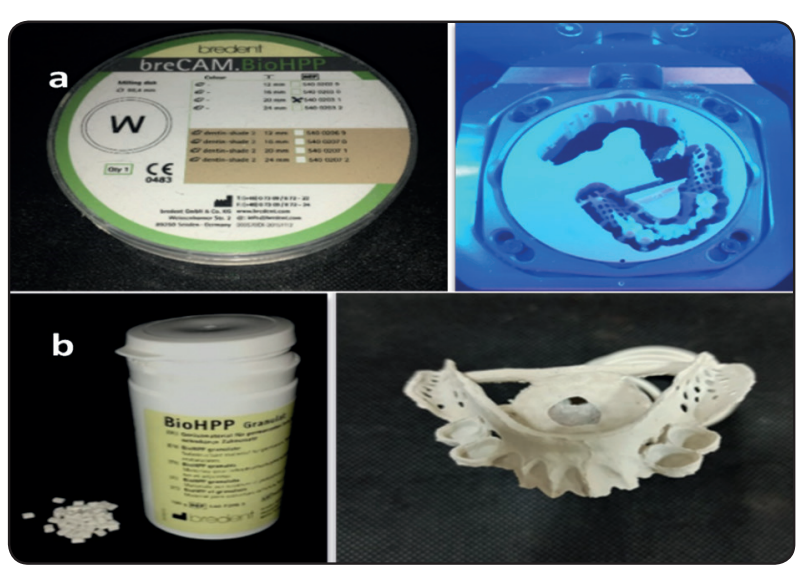

Fig. (2) Milled BioHpp telescopic partial denture

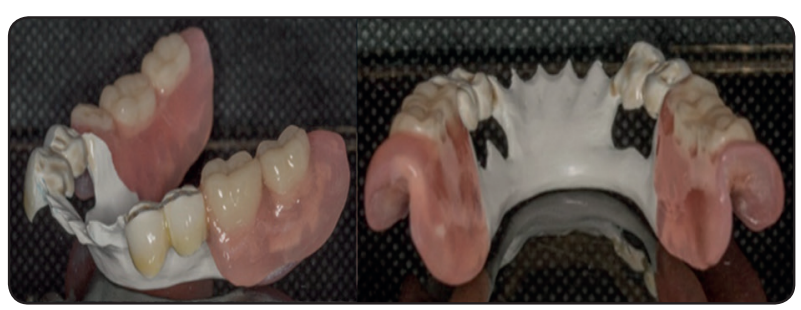

Fig. (3): pressed BioHpp telescopic partial denture

\section{h) Follow-up visits and radiographic evaluation}

In the recall visits, patients were evaluated radiographically to measure the bone height changes around the natural abutments and the residual ridge of the distal extension. Measurements were taken at time of insertion, six months, and twelve months post-insertion. The phosphor plate film, the Rinn-XCP periapical film holder, and a specially constructed acrylic template were used for taking standardized and reproducible serial of digital images for the abutments and the residual ridge using the periapical long cone paralleling technique. Radiographic stent fabrication:

A light-cured acrylic stent was constructed over an obtained cast to cover the area over the abutments and the distal extension edentulous area. Before polymerization, the bite block of the film holder was placed on the stent to create its place, then the whole assembly was polymerized in the light cure device.

\section{Image analysis:}

The images were inspected, and the bone height changes were assessed using the linear measuring tools in the software system (Soredex Digora Optime).

\section{Marginal bone level measurements:}

For standardization, the digital images were analysed as followed:

i) First line was drawn tangential to the apex of the abutment (line "1") parallel to the horizontal plane and perpendicular to the tooth long axis.

ii) Two other lines were drawn. (line " 2 " and line " 3 "), one on the mesial of the first abutment and the other on the distal of the second abutment and extended from the highest level of the marginal bone to the horizontal line (line "1").

\section{Residual ridge bone height measurement:}

i) Another tangential line (line "4") was drawn extending for $15 \mathrm{~mm}$ from the root apex of 
the last abutment and parallel to the horizontal plane, this distance was standardized with all patients.

ii) A line (line " 5 ") was then drawn ascending till the crest of residual ridge from (line " 4 ") and parallel to the tooth long axis.

\section{RESULTS}

Statistical analysis Data were collected, tabulated, and statistically analysed using Microsoft Excel ${ }^{\circledR}$ 2016 (Microsoft Cooperation, USA) , Statistical Package for Social Science (SPSS) ${ }^{\circledR}$ Ver. 24 (IBM Product, USA), and Minitab ${ }^{\circledR}$ statistical software Ver. 16 (IBM Product, USA). Data were revealed as mean difference $(\mathrm{mm})$ and standard deviation. Comparison between the two groups in all intervals was performed by using Student's t-test.
- Comparison between milled BioHpp (group I) and pressed BioHpp (group II) telescopic removable partial dentures:

The mean difference, standard deviation, and $\mathrm{P}$ value of the Student's t-test of the bone height changes throughout the time from insertion to twelve months post insertion and their level of significance is presented in table (I) and figure (4). Group II (pressed BioHpp) showed lower bone loss around the abutments than group I (milled BioHpp), by using Student's-t-test this difference was statistically significant, however, regarding the distal extension part of the ridge, group II (pressed BioHpp) showed higher bone loss than group I (milled BioHpp) and this was statistically significant after using the Student's t-test.

TABLE (I): Mean difference, SD, and P value of Student's t-test test for comparison between both groups throughout the different intervals:

\begin{tabular}{|c|c|c|c|c|c|c|}
\hline \multirow{2}{*}{\multicolumn{2}{|c|}{$\begin{array}{c}\text { Time interval } \\
\text { Mean }\end{array}$}} & \multicolumn{2}{|c|}{ Group I } & \multicolumn{2}{|c|}{ Group II } & \multirow{3}{*}{\begin{tabular}{|c} 
Pvalue \\
$0.0005^{*}$
\end{tabular}} \\
\hline & & \multirow{2}{*}{$\frac{\text { SD }}{0.16}$} & \multirow{2}{*}{$\begin{array}{c}\text { Mean } \\
0.03\end{array}$} & \multirow{2}{*}{$\frac{\text { SD }}{0.1}$} & \multirow[b]{2}{*}{0.02} & \\
\hline$=$ & Mesial of $1^{\text {st }}$ Abutments & & & & & \\
\hline$\overline{\mathfrak{B}}$ & Distal of $2^{\text {nd }}$ abutments & 0.49 & 0.07 & 0.3 & 0.05 & $0.0001 *$ \\
\hline '́ & Overall & 0.32 & 0.05 & 0.2 & 0.03 & $0.0001 *$ \\
\hline $\mathbf{N}$ & Distal extension & 0.15 & 0.02 & 0.3 & 0.04 & $0.0001 *$ \\
\hline & Mesial of $1^{\text {st }}$ Abutments & 0.25 & 0.04 & 0.19 & 0.03 & $0.008 *$ \\
\hline $\overrightarrow{0}$ & Distal of $2^{\text {nd }}$ abutments & 0.85 & 0.13 & 0.31 & 0.05 & $0.0001^{*}$ \\
\hline $\mathfrak{I}$ & Overall & 0.55 & 0.08 & 0.25 & 0.04 & $0.0001^{*}$ \\
\hline 0 & Distal extension & 0.41 & 0.02 & 0.45 & 0.05 & 1.000 \\
\hline ? & Mesial of $1^{\text {st }}$ Abutments & 0.41 & 0.06 & 0.29 & 0.04 & $0.0017 *$ \\
\hline $\bar{g}$ & Distal of $2^{\text {nd }}$ abutments & 1.34 & 0.2 & 0.61 & 0.09 & $0.0001 *$ \\
\hline 7 & Overall & 0.87 & 0.13 & 0.45 & 0.07 & $0.0001 *$ \\
\hline$\overline{\mathbb{N}}$ & Distal extension & 0.56 & 0.08 & 0.75 & 0.11 & $0.003 *$ \\
\hline
\end{tabular}

\section{M; Mean diff. $\quad$ SD; standard deviation $\quad *$ Significant difference}




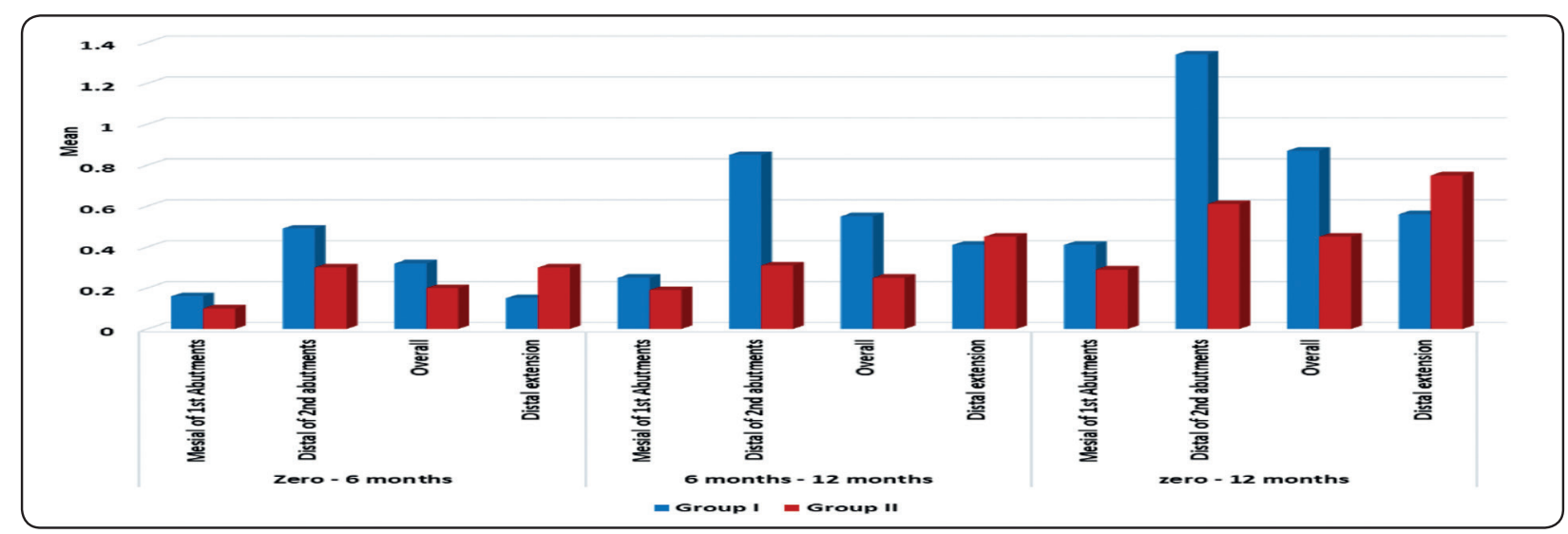

Fig. (4): Bar chart represents comparison between group I \& II in all intervals.

\section{DISCUSSION}

Patients with mandibular bilateral distal extension edentulous areas were chosen in this research. This condition is the most encountered in partially edentulous cases, and complications occur in such cases due to the nature of the mucosa that supports the denture base and the small denture-bearing region in the mandible. Tooth-tissue supported dentures are exposed to forces acting along three axes. These harmful forces are continuously applied horizontally, obliquely, and vertically causing ridge reduction. ${ }^{(10)}$

In this study, CAD / CAM technology was employed to create the telescopic removable partial dentures. This approach provides several advantages, including increased fitting precision, increasing production speed, fewer laboratory processes, and lower error factors. Another advantage of this manufacturing procedure is the possibility of digitally storing the patient's data and images for future replacement in the event the patient loses or damages his prosthesis. ${ }^{(11)}$

Recently, zirconia and PEEK have been proposed as tooth-coloured telescopic attachments, but rare clinical studies are available about their behaviour when combined and their effect on the supporting structures of the natural abutments, therefore it was recommended to perform more clinical research to assess the behaviour of these materials when placed opposite to each other under function in the oral cavity. ${ }^{(12,13)}$

The BioHpp frameworks were produced using its two recognised methods: the milled (from BioHpp blanks) and the pressed (from BioHpp granules). Studies have shown that both factors (the mode of manufacture and the preformed shape of the BioHpp) can affect the mode of retention with the primary crown and the flexural strength of the framework. ${ }^{(7,14)}$ Thus, the objective of this study was to determine the effect of the mode of production of the BioHpp on the supporting structures of the abutments and the residual ridge.

Clinically, both types of the BioHpp telescopic RPDs provided acceptable results in terms of retention, light weight and post-insertion adjustments, although the milled BioHpp showed better retention. Technically, the milled BioHpp was easier in fabrication than the pressed BioHpp.

Despite the regulatory oral and denture hygiene measures, the reported crestal bone loss in this study was within the acceptable range which may be attributed to presence of telescope retainers that transmit occlusal forces in a favourable direction, as they transmit the occlusal forces in the direction of the long axes of the supporting teeth. Also, the splinting of the abutments has favoured the situation 
and the amount of bone resorption around the abutments. ${ }^{(15,16)}$

The results of this study showed that in both groups the last abutments adjacent to the distal extension area are the most affected, especially their distal surfaces. This finding could be explained by the fact that when occlusal forces are applied to the distal-extension RPDs, the abutments are mildly displaced, with greater movements in the direction of soft tissues due to lack of posterior support, causing tensile forces on the abutments in distal direction. This came in line with the findings of a study that indicated that the highest strain values obtained from the strain gauges were distal to the terminal abutments. Telescopic removable partial dentures in distal extension cases transfer most of the occlusal load to the distal surface of the last abutments and this problem occurs mostly in the mandible since it has less supporting tissue. ${ }^{(17)}$

Regarding bone height loss around the abutments, the results of this study showed a statistically significant bone resorption around the abutments in group I (milled BioHpp) more than in group II (pressed BioHpp). This could be due to the more stresses that is carried out by the abutments in group I due to the more adaptation and retention observed in this group.

There is a direct association between the magnitude of stress and torque transferred to the supporting structures and the amount of retention provided by the attachment, also, it was previously noted that the amount of bone resorption that occurs after denture insertion can be influenced by a variety of factors including the amount of load transmitted, the type of the prosthesis, the bone quality, the opposing arch and the patient age and gender. ${ }^{(18,19)}$

It was observed throughout the current study follow-up period that group I (milled BioHpp) showed better retention than group II (pressed BioHpp), and this conforms with a recent studies that found that the retention force of milled telescopic crowns had higher values than the ones produced by the lost wax technique, also, it maintained its retentive values for a longer period. ${ }^{(20,21)}$

This observation could be explained by the fact that the pressed BioHpp as a technique is similar to the conventional lost wax technique, which includes more complicated sequence of steps with a greater number of potential sources of error, in particular, the unpredictable coefficient of expansion of the investment material caused dimensional changes, the fitting values at the inner surface is affected by the contraction of the material during cooling. Roughening of the inner surface by airborne particles to remove the investment material, also might have affected the retention forces. The milled BioHpp, on the other hand, is a complete CAD/ CAM fabrication process that is only influenced by the parameters of the software program, and such difference in mode of fabrication could affect the precision and retention between the primary and secondary copings. ${ }^{(7,20,21)}$

Another reason could be the softer nature inherited in the pressed Biohpp telescopic frameworks which added to its resiliency in comparison to the milled BioHpp telescopic frameworks, this might have led to a more stressbreaking action between the telescopic crowns and the denture base, consequently, more load distribution between the abutments and the residual ridge. It was found in a recent study that the preform shape of the BioHpp (blanks, pellets, or granules) affects the flexibility and mechanical behaviour of the final BioHpp product. The study stated that the BioHpp pressed from the granular form is softer and has lower modulus of elasticity unlike the BioHpp blanks because industrially, the granular form passes no prepressing, while the BioHpp blanks and pellets passes through a prepressing process and are extruded out from the BioHpp granules which is the raw material of both the blanks and the pellets, this 
increases their mechanical properties in comparison to the granular form. ${ }^{(14)}$

Regarding the residual ridge crestal bone resorption in the distal extension area, the results of this study showed that in group II (pressed BioHpp) there was a statistically significant increase in the amount of bone reduction than in group I (milled BioHpp). Again, this could be due to the more elasticity encountered within the pressed BioHpp telescopic frameworks that led to extra tissue-ward movement of the denture bases during function. Also, the improved retention in group I (milled BioHpp) telescopic RPDs most probably minimized the denture base movement posteriorly.

\section{CONCLUSION}

Within the limitation of this study, it could be concluded that, milled and pressed Bio-Hpp telescopic partial denture produced acceptable treatment options regarding bone loss. Milled BioHpp frameworks (group I) had a greater effect on bone resorption around the abutments than pressed BioHpp (group II) while at the residual ridge area, pressed BioHpp showed more bone height changes than milled one.

\section{REFERENCES}

1. Carr AB, Brown DT. McCracken's removable partial prosthodontics. 13 ed. Elsevier Health Sciences; 2015. p 21-28.

2. Stawarczyk B, Ender A, Trottmann A, Özcan M, Fischer $\mathrm{J}$, Hämmerle CHF. Load-bearing capacity of CAD/CAM milled polymeric three-unit fixed dental prostheses: Effect of aging regimens. Clin Oral Investig. 2012 Dec 3;16(6):1669-77.

3. Schwindling FS, Dittmann B, Rammelsberg P. Doublecrown-retained removable dental prostheses: A retrospective study of survival and complications. The Journal of prosthetic dentistry. 2014 Sep 1;112(3):488-93.

4. Brenes C, Duqum I, Mendonza G. Materials and systems for all ceramic CAD/CAM restorations. Dental Tribune. CAD/CAM international magazine of digital dentistry. 2016;3:10-5.
5. Tekin S, Cangül S, Adıgüzel Ö, Değer Y. Areas for use of PEEK material in dentistry. International Dental Research. 2018 Aug 27;8(2):84-92.

6. Bredent. The new class of materials. www.bredent.com. 2016;5,6.

7. Merk S, Wagner C, Stock V, Eichberger M, Schmidlin PR, Roos M, Stawarczyk B. Suitability of secondary PEEK telescopic crowns on zirconia primary crowns: the influence of fabrication method and taper. Materials. 2016 Nov;9(11):908.

8. Emera RM, Altonbary GY, Elbashir SA. Comparison between all zirconia, all PEEK, and zirconia-PEEK telescopic attachments for two implants retained mandibular complete overdentures: In vitro stress analysis study. Journal of Dental Implants. 2019 Jan 1;9(1):24.

9. Schubert O, Reitmaier J, Schweiger J, Erdelt K, Güth JF. Retentive force of PEEK secondary crowns on zirconia primary crowns over time. Clinical oral investigations. 2019 May;23(5):2331-8.

10. Rodney D. Phoenix, David R. Cagna CFD. Stewart's Clinical Removable Partial Prosthodontics. 4th ed. Quintessence Publishing Co, Inc; 2008. 104-105 p.

11. Bohnenkamp DM. Removable partial dentures: clinical concepts. Dental Clinics of North America. 2014 Jan $1 ; 58(1): 69-89$

12. Hakkoum MA, Wazir G. Telescopic Denture. Open Dent J. 2018;12(1):246-54.

13. M. M. S. Rahhal AEF. A systematic review studying the effect of different framework materials on the retention of implant telescopic overdentures. Int $\mathrm{J}$ Adv Res. 2017;5(2):2627-40.

14. Stawarczyk B, Eichberger M, Uhrenbacher J, Wimmer T, Edelhoff D, Schmidlin PR. Three-unit reinforced polyetheretherketone composite FDPs: influence of fabrication method on load-bearing capacity and failure types. Dental materials journal. 2015 Jan 30;34(1):7-12.

15. Veena Hegde, Shashi Rashmi Acharya GPS. Validity of Crown-To-Root Ratio as a Prognostic Tool in Clinical Practice: A Review Article. J Evol Med Dent Sci. 2014;3(74):15589-99.

16. Goswami R, Mahajan P, Siwach A, Gupta A. Telescopic overdenture: Perio-prostho concern for advanced periodontitis. Contemporary clinical dentistry. 2013 Jul;4(3):402. 
17. Sahin V, Akaltan F, Parnas L. Effects of the type and rigidity of the retainer and the number of abutting teeth on stress distribution of telescopic-retained removable partial dentures. J Dent Sci. 2012;7(1):7-13.

18. Chikunov I, Doan P, Vahidi F. Implant-retained partial overdenture with resilient attachments. Journal of Prosthodontics. 2008 Feb;17(2):141-8.

19. Dyeus M Chung, Tae-Ju Oh, Jungwha Lee, Carl E Misch H-LW. Factors affecting late implant bone loss: A retrospective analysis. J Prosthet Dent. 2007;98(3):215.

20. Stock V, Wagner C, Merk S, Roos M, Schmidlin PR, Eichberger M, Stawarczyk B. Retention force of differently fabricated telescopic PEEK crowns with different tapers. Dental materials journal. 2016 Jul 29;35(4):594-600.

21. Luft V, Pospiech P, Schurig A, Schmitter M. In vitro investigations on retention force behavior of conventional and modern double crown systems. Dental Materials. 2021 Jan 1;37(1):191-200. 